\title{
Reconstrucción con bloque óseo tricortical cadavérico en luxación glenohumeral anterior recurrente con pérdida ósea glenoidea: técnica modificada de Eden Hybinette
}

\author{
Reconstruction with cadaveric tricortical bone block in recurrent anterior glenohumeral \\ dislocation with glenoid bone loss: modified Eden Hybinette technique
}

Martínez-Montiel O,* Valencia-Martínez G, ${ }^{\ddagger}$ Jasso-Ramírez LA ${ }^{\S}$

Centro Médico ISSEMyM, Toluca.

RESUMEN. La luxación glenohumeral recurrente suele asociarse a pérdida ósea de la porción glenoidea, siendo la localización anteroinferior la más afectada. El entendimiento de las estructuras relacionadas así como el uso de estudios de imagen actuales tales como la tomografía axial computarizada y la resonancia magnética han permitido avanzar respecto a la comprensión de la patología, asimismo el desarrollo de materiales quirúrgicos y herramientas de mínima invasión nos permiten continuar innovando respecto a los tratamientos previamente descritos, siendo posible intervenir en detalles técnicos con la intención de mejorar los resultados. Es por eso que hemos realizado lo descrito por Eden-Hybinette utilizando injerto tricortical cadavérico con tornillos canulados como método de fijación, limitando las comorbilidades asociadas a la toma de autoinjerto, dando como resultado un amplio beneficio para el paciente durante el procedimiento quirúrgico y en el período de recuperación.

Palabras clave: Luxación, hombro, recurrente, tratamiento, bloque óseo.

\section{Nivel de evidencia: $\mathrm{V}$}

* Médico adscrito al Servicio de Ortopedia y Cirugía Articular.

¥ Jefe de Servicio Ortopedia y Cirugía Articular, Centro Médico ISSEMyM, Toluca.

${ }^{\S}$ Residente de Ortopedia cuarto año, Centro Médico ISSEMyM, Toluca.

Dirección para correspondencia: Octavio Martínez-Montiel

Tel: 7223566196

E-mail: dr.octaviomontiel@hotmail.com

https://dx.doi.org/10.35366/95326

doi: $10.35366 / 95326$
ABSTRACT: Recurrent glenohumeral dislocation is usually associated with bone loss of the glenoid portion, with the anteroinferior location being the most affected. The understanding of the related structures, as well as the use of current imaging studies such as computed axial tomography and magnetic resonance imaging, have made progress in understanding the pathology, as well as the development of surgical materials and minimally invasive tools, they allow us to continue innovating with respect to the previously described treatments, being possible to intervene in technical details with the intention of improving the results. That is why we have done what described by Eden-Hybinette, using tricortical cadaveric graft and as fixation method, limiting the comorbidities associated with the autograft taking, resulting a wide benefit for the patient, during the surgical procedure and in the recovery period.

Keywords: Dislocation, shoulder, recurring, treatment, bone block.

\section{Introducción}

El tratamiento de la inestabilidad glenohumeral asociada a pérdida ósea glenoidea ha sido motivo de debate durante las últimas décadas. El estudio comparativo entre técnicas quirúrgicas anatómicas y no anatómicas ha permitido conocer las indicaciones precisas ante la misma patología. ${ }^{1}$ Burkhart y colaboradores reportaron mayor índice de luxación en pacientes con pérdida ósea glenoidea > 25\% al realizar la técnica artroscópica de Bankart, por lo que en este tipo de lesiones prefieren una técnica de aumentación ósea de la glena. ${ }^{2,3}$ Una de las técnicas de aumento de superficie de la glenoides es la técnica de Latarjet, en un inicio fue descrita en 1954, consiste en una osteotomía de la coracoides y posteriormente una fijación longitudinal en la región anterior de la glenoides. A partir de entonces ha tenido diversas modificaciones al grado que hoy en día se puede ha- 
cer de forma abierta, artroscópica y con diferentes métodos de fijación del injerto óseo. Las variantes de las técnicas y la habilidad del cirujano influyen en la posibilidad de recurrencia; sin embargo, se reporta un aproximado de 1 a $7 \%$ según diferentes estudios. 4,5,6,7,8

Para estos casos de recurrencia una de las propuestas es la técnica de Eden Hybinette, ${ }^{9}$ la cual fue descrita como un aumento de la superficie glenoidea a partir de la colocación de injerto óseo tomado de la cresta ilíaca. Otras de las variantes de esta técnica es el uso de injerto tomado de la espina tibial y también con modificaciones con respecto al tipo de fijación. Se ha abordado esta técnica como un tratamiento primario, ya que se ha reportado mayor índice de artritis glenohumeral, lisis y procedimientos de no unión del injerto. ${ }^{10,11}$

Tanto la técnica de Eden Hybinette como la descrita por Latarjet tienen pros y contras. En el caso de la primera opción al sólo realizarse una transferencia ósea hay una pérdida del efecto columpio que ofrece Latarjet; sin embargo, se han descrito estudios en los cuales esto no refleja una superioridad con respecto a la recurrencia de luxaciones. De igual forma es más común tener una falla al realizar la osteotomía de la coracoides y que se tome un injerto insuficiente para la fijación ósea o incluso fracturas de la misma. En ambas técnicas la toma del injerto puede resultar en complicaciones anexas que pueden evitarse. Es por esto que hemos desarrollado una modificación a la técnica descrita por Eden Hybinette usando injerto heterólogo de cadáver con fijación ósea con tornillos canulados. Con esta técnica podemos obtener una disminución en la falla en la toma de injerto, del dolor postoperatorio, del tiempo quirúrgico y una mayor satisfacción del paciente.

Se coloca al paciente en posición silla de playa con el tronco en flexión de $30^{\circ}$, se coloca soporte cervical rígido como protección de la columna cervical (Figura 1); asimismo, se precisa apoyo de un soporte de antebrazo previo a la realización de portales artroscópicos.

A través de un portal posterior se ingresa a la articulación glenohumeral, mediante visión directa se inicia recorrido sistemático identificando lesiones estructurales asociadas que contribuyen a la inestabilidad, se corrobora pérdida

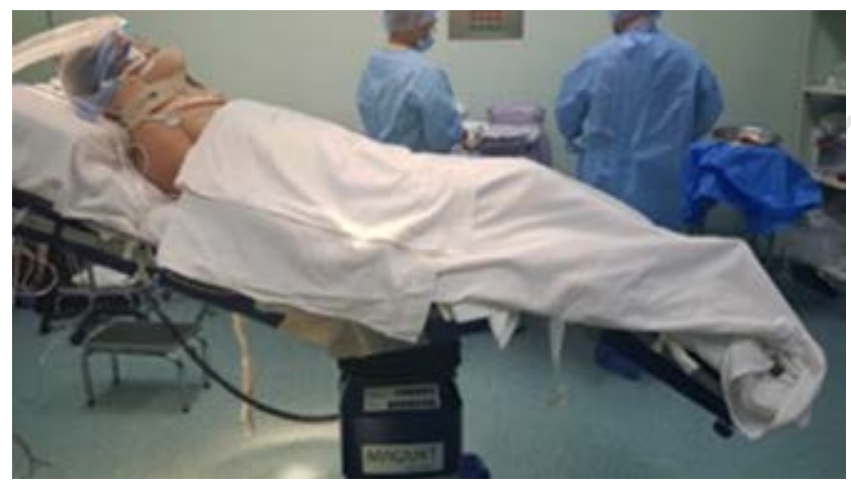

Figura 1: Posición de paciente.

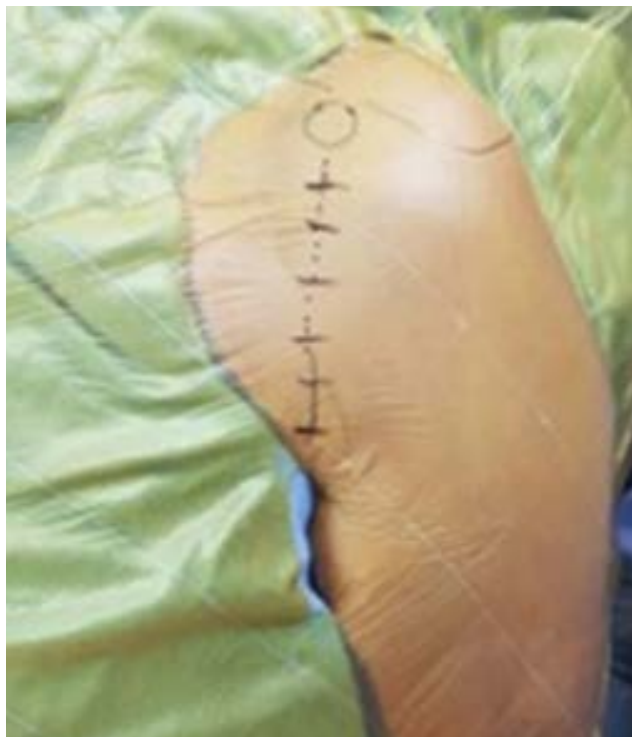

Figura 2:

Sitio de incisión.
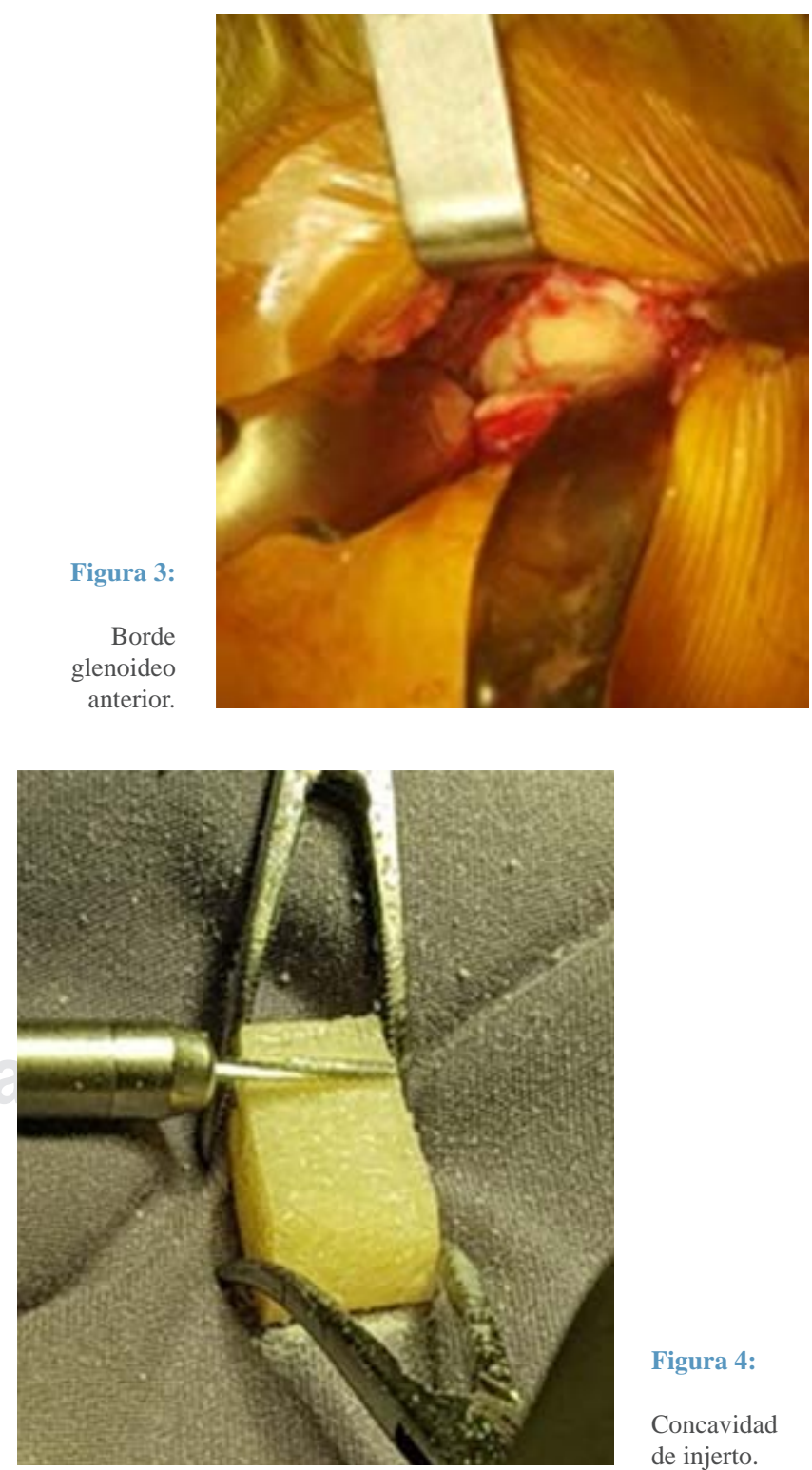

Figura 4:

Concavidad de injerto. 
ósea glenoidea y se hace una evaluación de las estructuras musculares y tendón de porción larga del bíceps, con técnica fuera dentro se realiza portal anterosuperior, se procede a realizar reparaciones de lesiones adicionales (labrum o cápsula articular). Posterior a la artroscopía se efectúa un abordaje deltopectoral, se toma como referencia anatómica la apófisis coracoides y el surco deltopectoral; a continuación se hace una incisión de aproximadamente $5 \mathrm{~cm}$ estableciendo como límite proximal la proyección inferolateral de la coracoides y siguiendo la proyección del surco deltopectoral (Figura 2). Se localiza plano intermuscular en el intervalo del pectoral mayor y deltoides, rechazando medial y lateral respectivamente, se realiza retracción gentil hacia medial de la vena cefálica, preservándola en la medida de lo posible. Se localiza tendón conjunto de músculo coracobraquial y
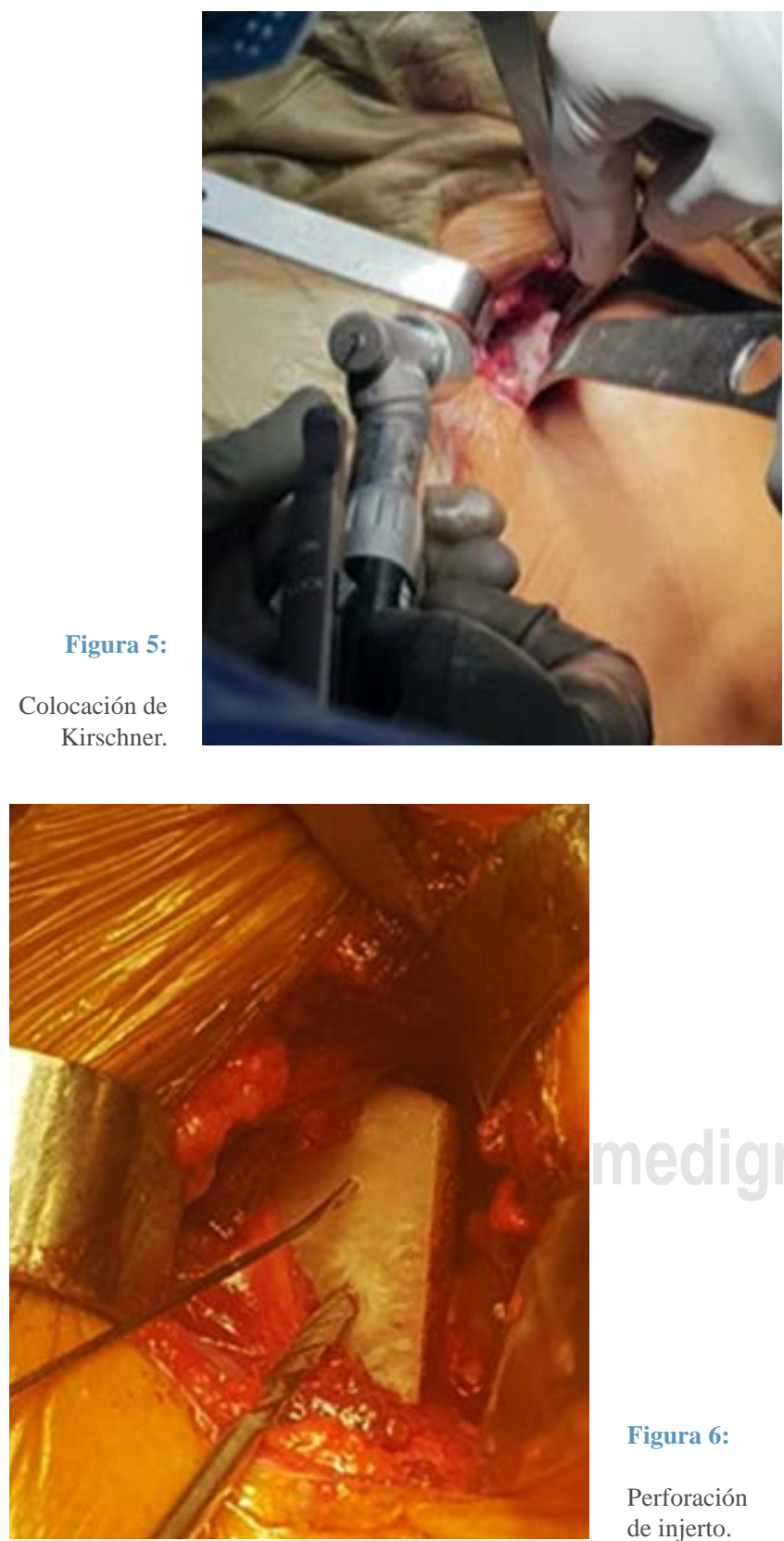

Figura 6:

Perforación de injerto.
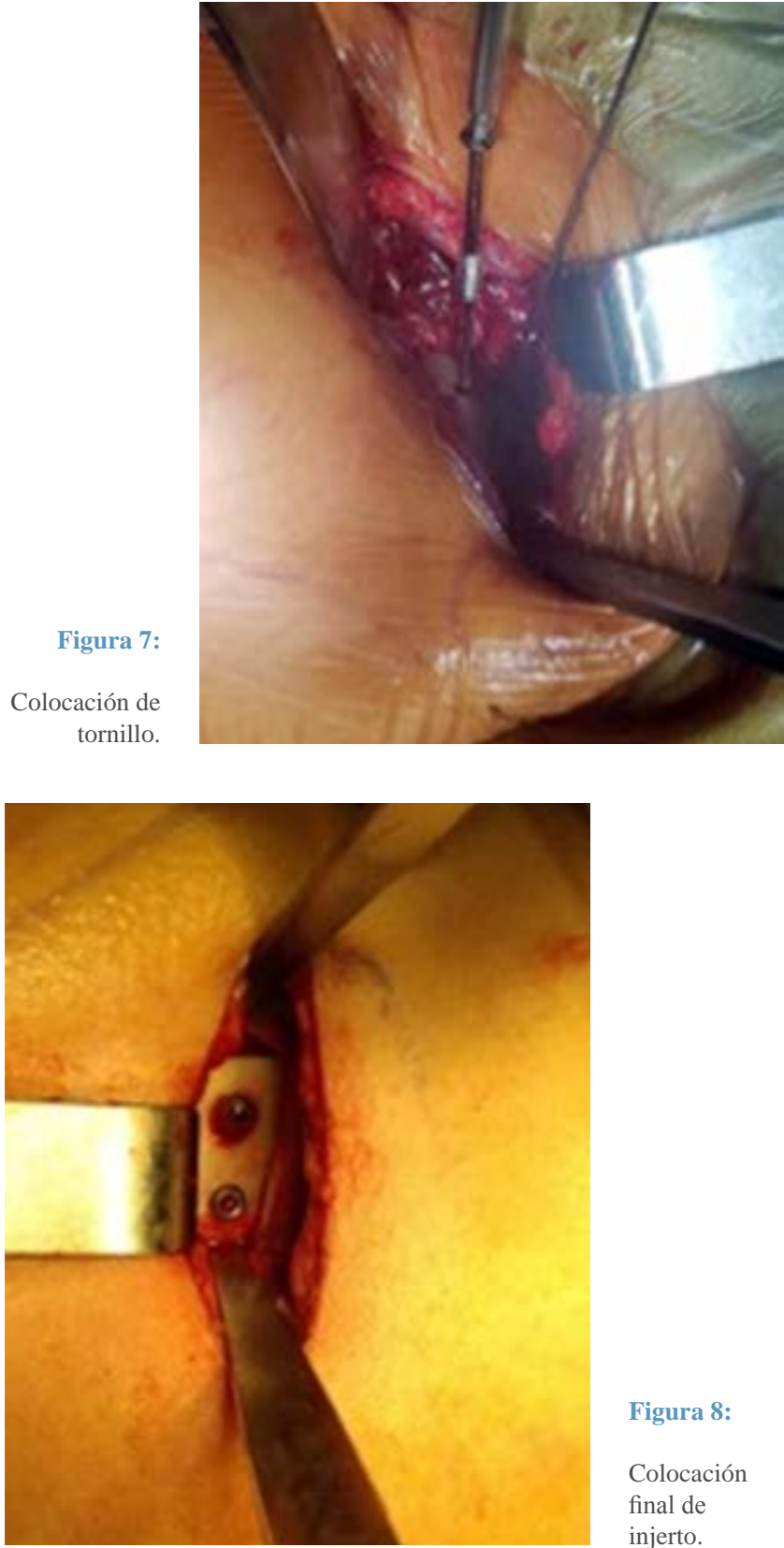

Figura 8:

Colocación final de injerto.

porción corta de bíceps braquial, el cual servirá como límite medial durante el procedimiento. Se hace disección roma en sentido longitudinal del músculo subescapular para acceder a porción anterior extraarticular de la glenoides. Se realiza una capsulotomía longitudinal en dirección céfalo-caudal, refiriendo los bordes capsulares localizando pérdida ósea glenoidea (Figura 3); se realiza desperiostización de la porción anterior de la glenoides con fresado con la intención de promover el sangrado local y el contacto directo de hueso sangrante con el aloinjerto.

Se utiliza injerto óseo cadavérico de cresta ilíaca liofilizado con medidas previamente determinadas por tomografía. Previo a la colocación se realiza corte con sierra de mano, creando una superficie cóncava que resulte congruente con el borde glenoideo anterior (Figura 4). Se retira exceso de 
hueso creando una forma rectangular. Previa preparación de porción glenoidea se coloca el injerto con ayuda de clavillos Kirschner $1.2 \mathrm{~mm}$ en porción superior e inferior de porción anterior de la glenoides, estableciendo la posición correcta (Figura 5), para la fijación estable se hace perforación con broca canulada $2.5 \mathrm{~mm}$ a través de injerto óseo y cuello glenoideo tomando tres corticales (Figura 6). Se colocan tornillos canulados $3.5 \mathrm{~mm}$ rosca discontinua a través de clavillos Kirschner (Figura 7). Por último se retiran ambos clavillos obteniendo la fijación definitiva (Figura 8). Se cierra la cápsula en su totalidad con Monocryl 2-0 con cabos previamente referidos. Se sutura músculo subescapular con puntos en $\mathrm{U}$, se colocan puntos de afrontamiento para plano muscular entre pectoral mayor y deltoides, por último se cierra la herida con sutura en tejido celular subcutáneo y piel.

\section{Bibliografía}

1. Balg F, Boileau P. The instability severity index score: a simple preoperative score to select patients for arthroscopic or open shoulder stabilisation. J Bone Joint Surg Br. 2007; 89(11): 1470-7.

2. Burkhart SS, De Beer JF. Traumatic glenohumeral bone defects and their relationship to failure of arthroscopic Bankart repairs: significance of the inverted-pear glenoid and the humeral engaging Hill-Sachs lesion. Arthroscopy. 2000; 16(7): 677-94.
3. Boileau P, Villalba M, Héry JY, Balg F, Ahrens P, Neyton L. Risk factors for recurrence of shoulder instability after arthroscopic Bankart repair. J Bone Joint Surg Am. 2006; 88(8): 1755-63.

4. Bessiere C, Trojani C, Carles M, Mehta SS, Boileau P. The open Latar- jet procedure is more reliable in terms of shoulder stability than arthroscopic Bankart repair. Clin Orthop Relat Res. 2014; 472(8): 2345-51.

5. Laurent W, Bassem E, Olivier V. Bony reconstruction of the glenoid rim. J Am Acad Othop Surg. 2018; 26(10): e207-e218.

6. Burkhart SS, De Beer JF, Barth JR, Cresswell T, Roberts C, Richards DP. Results of modified Latarjet reconstruction in patients with anteroinferior instability and significant bone loss. Arthroscopy. 2007; 23(10): 1033-41.

7. Rabinowitz J, Friedman R, Eichinger JK. Management of glenoid bone loss with anterior shoulder instability: indications and outcomes. Curr Rev Musculoskelet Med. 2017; 10(4): 452-62.

8. Lunn JV, Castellano-Rosa J, Walch G. Recurrent anterior dislocation after the Latarjet procedure: Outcome after revision using a modified Eden-Hybinette operation. J Shoulder Elbow Surg. 2008; 17(5): 744-50.

9. Hybinette S. De la transplantation d'un fragment osseux pour remédier aux luxations recidivantes de L’Epaule: constations et résultats operatoires. Acta Chir Scand. 1932; 71: 411-55.

10. Warner JJ, Gill TJ, O’Hollerhan JD, Pathare N, Millett PJ. Anatomical glenoid reconstruction for recurrent anterior glenohumeral instability with glenoid deficiency using an autogenous tricortical iliac crest bone graft. Am J Sports Med. 2006; 34(2): 205-12.

11. Ernstbrunner L, Plachel F, Heuberer P, Pauzenberger L, Moroder P, Resch H, et al. Arthroscopic versus open iliac crest bone grafting in recurrent anterior shoulder instability with glenoid bone loss: a computed tomography-based quantitative assessment. Arthroscopy. 2018; 34(2): 352-9. 